\title{
Evaluation of Surface Acoustic Wave Motor Stepping Motion
}

\author{
Takashi Shigematsu, Minoru Kuribayashi Kurosawa \\ Tokyo Institute of Technology, Department of Advanced Applied Electronics, \\ G2-606, 4259 Nagatsuta, Midori-ku, Yokohama 226-8502, Japan \\ Katsuhiko Asai \\ Matsushita Electric Industrial Co., Ltd., Advanced Technology Research Laboratories, \\ 3-4 Hikaridai, Seika, Souraku, Kyoto 619-0237, Japan
}

\begin{abstract}
Utilizing nanometer vibration of surface acoustic wave for friction drive enables nanometer stepping drives of a surface acoustic wave motor. Number of driving waves varied step displacement readily, and decrease of driving waves brought about 2 $\mathrm{nm}$ step motion as a minimum step of an experimental setup. We proposed a simulation model so as to control step displacement at open loop condition. The simulation model represented the characteristic transient response of the surface acoustic wave motor, and it can be applied to an open loop control of a rigid structural surface acoustic wave motor. However, the experimental setup did not have enough rigidity. Accurate simulation of the experimental setup requires taking elastic deformations into consideration. We, therefore, proposed a mechanical equivalent circuit model that takes accounts of compliance of the structure.
\end{abstract}

\section{INTRODUCTION}

Surface acoustic wave motor is a kind of ultrasonic motor that uses RF Rayleigh wave for friction drive. Conventional ultrasonic motors, which use micrometer order vibration for friction drive, have sub-micrometer positioning [1]. Surface acoustic wave motor uses nanometer order vibration for friction drive. On the analogy of conventional ultrasonic motors, we have suggested surface acoustic wave motor's possibility of nanometer order stepping drive [2]. Due to the technical limit of measuring equipments, we have only reported $40 \mathrm{~nm}$ [3] and $25 \mathrm{~nm}$ [4] stepping drives. This paper describes nanometer stepping drives of surface acoustic wave motor measured with a laser Doppler vibrometer that has $2 \mathrm{pm}$ resolution.

For industrial application, control system on this

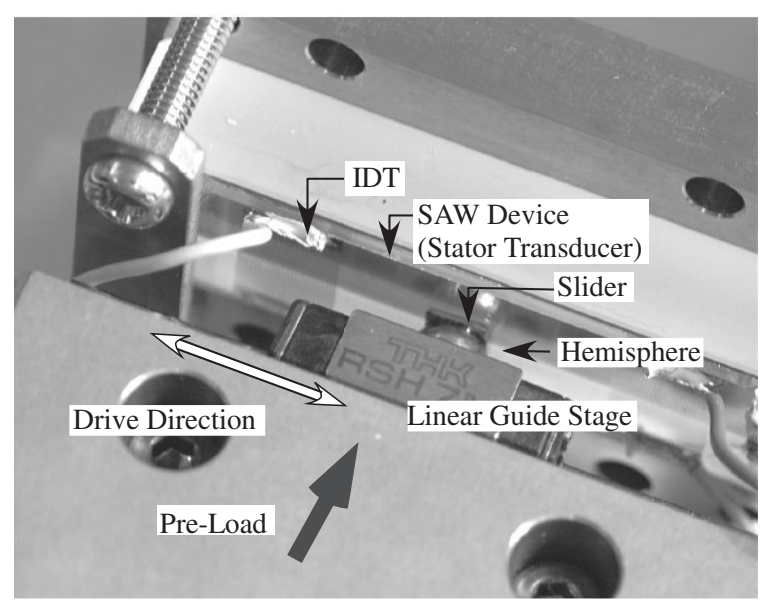

Fig. 1. Photograph of the experimental setup.

high resolution positioning motor has important meanings. However, any positioning control has never applied to surface acoustic wave motor. We propose a simple simulation model in this paper for precision positioning control. The simulation model enables to calculate the step displacement by integration of temporal change of output force.

\section{EXPERIMENTAL SETUP}

An experimental setup is shown in Fig.1. The setup consisted of a fixed part and a movable part. A 60x15x1 $\mathrm{mm}^{3}$ stator transducer and a linear guide rail were the fixed part, and they were fixed in parallel. At each end of the startor transducer, interdigital transducers (IDTs) were deposited. The dimensions of the IDT were 400 $\mu \mathrm{m}$ in pitch, $100 \mu \mathrm{m}$ in electrode strip width and $9 \mathrm{~mm}$ in aperture. The IDT was composed of 20 strip electrode pairs and the resonance frequency for Rayleigh wave propagation was $9.6 \mathrm{MHz}$.

A movable part comprised a $4 \times 4 \times 0.3 \mathrm{~mm}^{3}$ silicon 


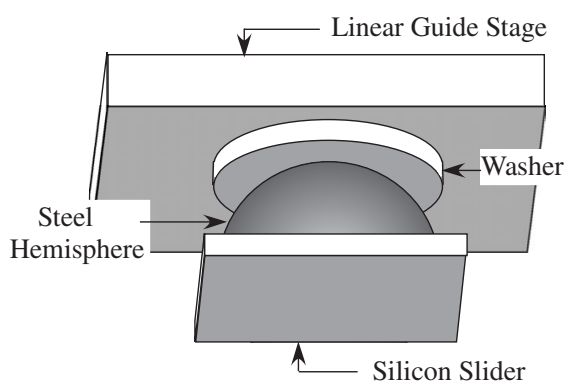

Fig. 2. Schema of the movable part of the setup.

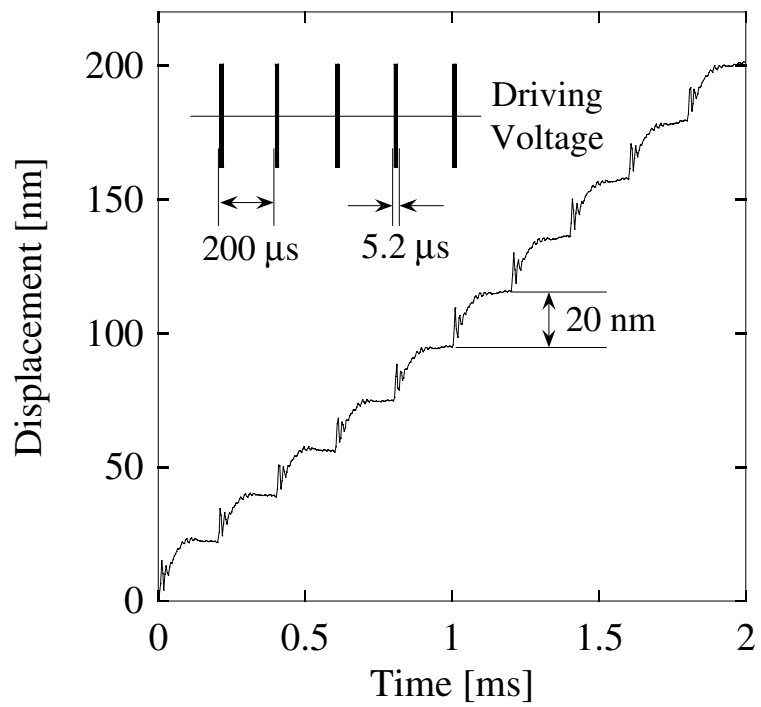

Fig. 3. The stepping drive motion; the driving voltage was $100 V_{\text {peak }}$, and the driving waves were $50 \mathrm{cy}$ cles, and the pre-load was $30 \mathrm{~N}$. The burst period was $0.2 \mathrm{~ms}$.

slider [5], a steel hemisphere and a linear guide stage. The movable part moved along the linear guide rail. The silicon slider was glued on the steel hemisphere. The steel hemisphere was connected with the linear guide stage so that the silicon slider contacted the stator transducer surface properly. The movable part is schematically illustrated in Fig.2. A laser beam of the laser Doppler vibrometer was incident on the steel hemisphere to measure the stepping motion of the silicon slider. The pre-load was given by a coil spring so that the pre-load adjusted easily.

\section{EXPERIMENTAL RESULTS}

Burst driving voltages were applied to the IDT every $0.2 \mathrm{~ms}$ to drive the surface acoustic wave motor

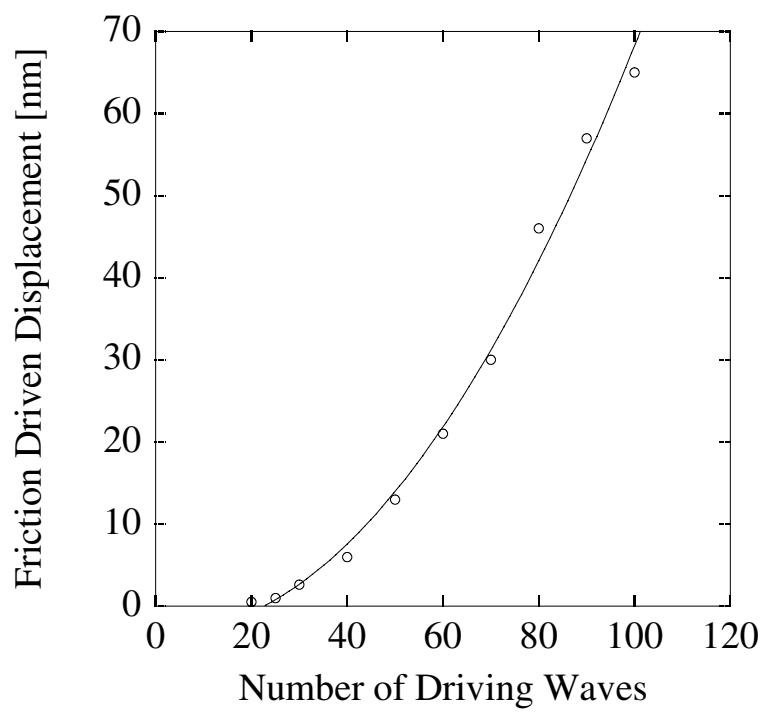

Fig. 4. Friction driven displacement against the number of driving waves; the driving voltage was 100 $V_{\text {peak }}$, and the pre-load was $30 \mathrm{~N}$.

in stepping motion. An example of stepping drive motion is shown in Fig.3, where the driving voltage was $100 \mathrm{~V}_{\text {peak }}$, the driving waves were 50 cycles, and the pre-load was 30 N. Step motions were observed every $0.2 \mathrm{~ms}$ when the driving voltages were applied. Each step displacement was about $20 \mathrm{~nm}$.

The friction driven displacements (mentioned later) were measured for estimation of the friction drive. The driving waves were varied from 20 cycles to 100 cycles, while the driving voltage was $100 \mathrm{~V}_{\text {peak }}$, and the pre-load was $30 \mathrm{~N}$. The friction driven displacement against the number of the driving waves is shown in Fig.4. The friction driven displacement increased with the square of the number of the driving waves. The $2 \mathrm{~nm}$ step motion was obtained at the 25 cycles of driving waves, as shown in Fig.5. It was the finest stepping drive motion of the experimental setup.

\section{Simulation Model}

The IDT on the stator transducer was composed of 20 strip electrode pairs. Then, 20 wave cycles were required for rise-up and fall-down of the amplitude of the Rayleigh wave, as schematically shown in Fig.6. $A$ denotes the steady state amplitude and $n$ denotes a wave count at the silicon slider as a parameter of time; the product of time and driving frequency $[n=$ $($ time $) \times\left(9.61 \times 10^{6}\right)$ ]. The amplitude of the Rayleigh 


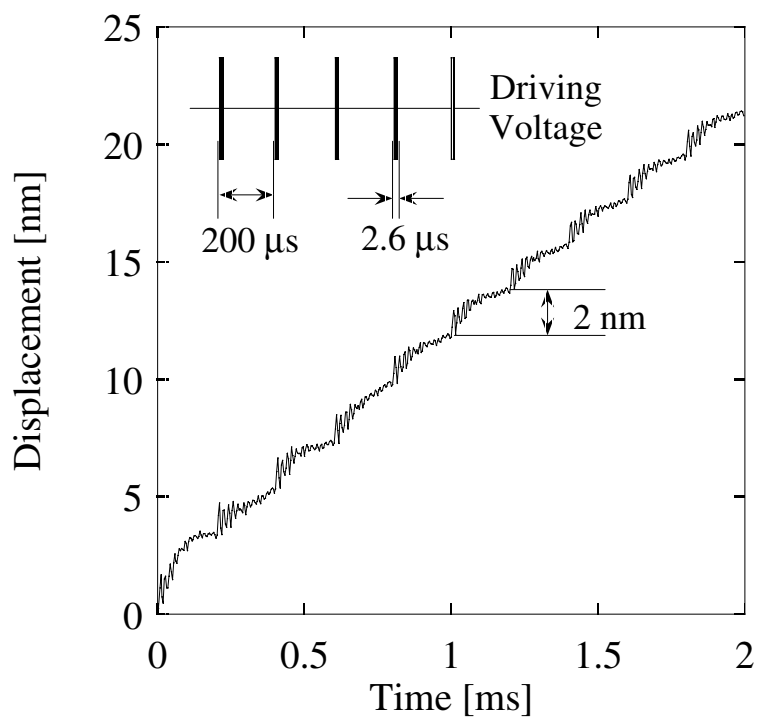

Fig. 5. The finest step, $2 \mathrm{~nm}$ step, motion of the experimental setup; the driving voltage was $100 V_{\text {peak }}$, the pre-load was $30 \mathrm{~N}$, and the driving waves were 25 cycles. The burst period was $0.2 \mathrm{~ms}$.

wave $A_{v}$ is written as

$$
\begin{array}{ll}
A_{v}=A \times \frac{n}{20} & (0 \leq n \leq 20) \\
A_{v}=A & (20 \leq n \leq N) \\
A_{v}=A\left\{1-\frac{n-N}{20}\right\} & (N \leq n \leq N+20)
\end{array}
$$

from Fig.6, where $N$ is the number of the applied driving waves to the IDT, and change of the amplitude of the Rayleigh wave assumes to be continuous.

There was a linear relation between the amplitude of the Rayleigh wave and the output force of the surface acoustic wave motor, as shown in Fig.7. The output force was calculated from the transient response of this motor [6]. The surface acoustic wave motor shows a typical transient response of ultrasonic motors; the speed rises as a first-order lag system (its step response has a form of $[1-\exp (-a t)])$ and falls linearly. Namely, the output force is maximum at the point where the slider starts moving. The driving time of the indicated experimental result was about $10 \mu \mathrm{s}$ (the driving waves were 100 cycles) at most, on the other hand, the rise-up time of the experimental setup was about $2 \mathrm{~ms}$. Since the driving time was much shorter than the rise-up time, about 1/200 at most, the reduction of the output force is assumed to be negligible at this period. The output force, thereby, can be

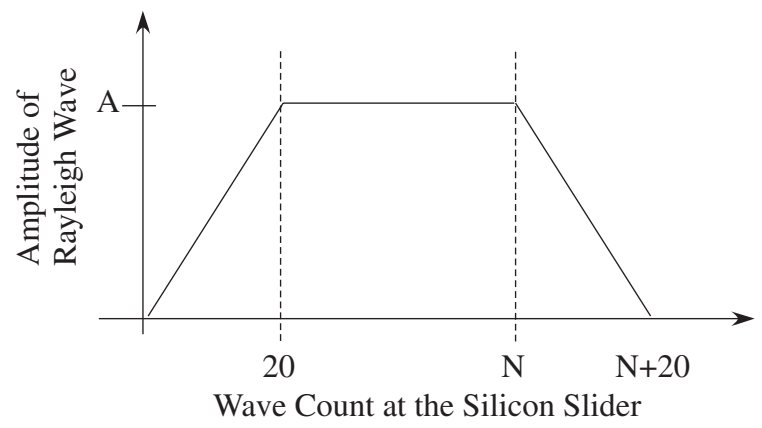

Fig. 6. Schematic view of the Rayleigh wave amplitude.

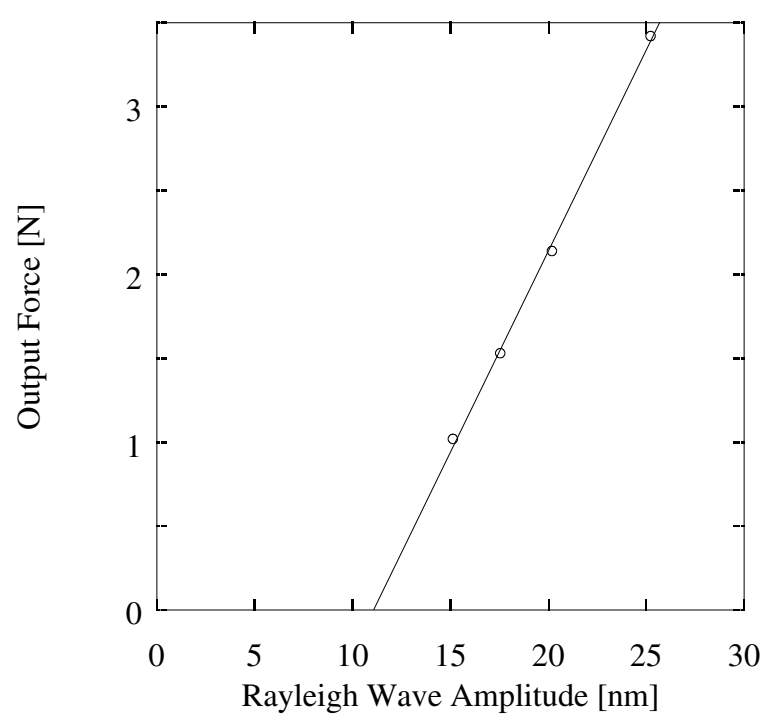

Fig. 7. Output force against the amplitude of steady state Rayleigh wave.

written as

$$
F=k A_{v}-r,
$$

where $k$ is the coefficient connecting the amplitude of the surface acoustic wave $A_{v}$ and the output force $F$, and $r$ is the force-axis intercepts of Fig.7.

Consequently, the acceleration is expressed as

$$
\begin{aligned}
a_{s} & =F / m \\
& =\left(k A_{v}-r\right) / m .
\end{aligned}
$$

After the Rayleigh wave has passed away, there is no driving force to the silicon slider, and the friction force exists. Hence, the acceleration at the period where the Rayleigh wave has passed away is expressed as

$$
a_{s}=-R / m
$$




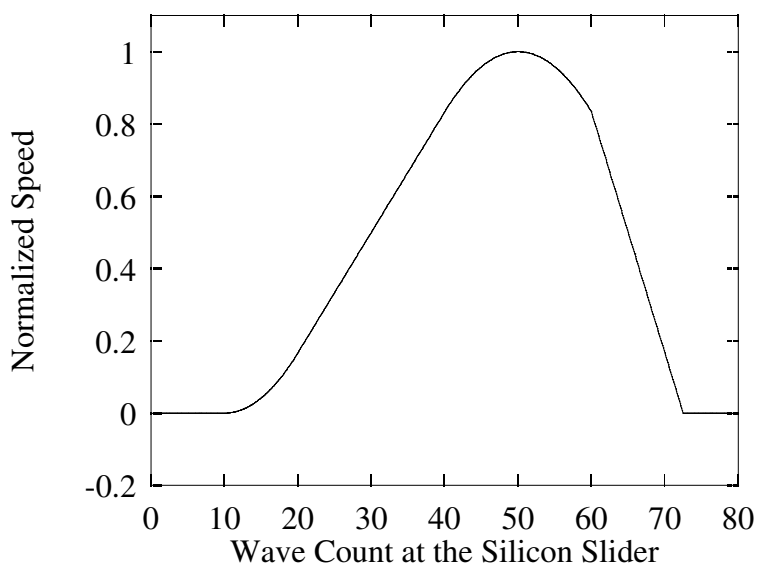

Fig. 8. The calculated speed normalized by the maximum speed; the driving waves were 40 cycles.

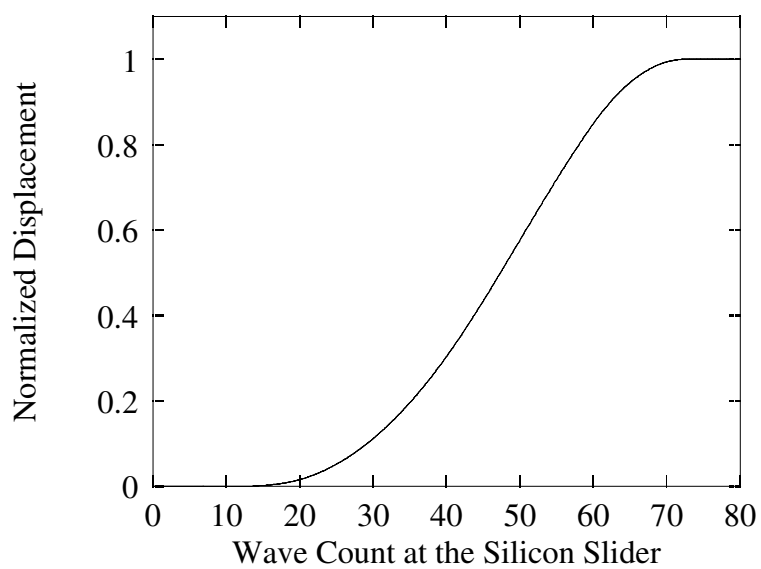

Fig. 9. The calculated displacement normalized by the maximum displacement; the driving waves were 40 cycles.

where $R$ indicates the friction force.

Simply integrating the equations (4) and (5), the speed and the displacement were calculated. Examples of the simulated speed and displacement are shown in Figs.8 and 9, where the driving waves $N$ were 40 cycles. Both the speed and the displacement were normalized by each maximum value.

\section{Evaluation}

The calculation results were compared with the experimental results while the driving voltage and the pre-load were maintained at $100 \mathrm{~V}_{\text {peak }}$ and $30 \mathrm{~N}$. From the relation of the output force and the Rayleigh wave amplitude indicated in Fig.7, the constants of equation (2) are estimated as

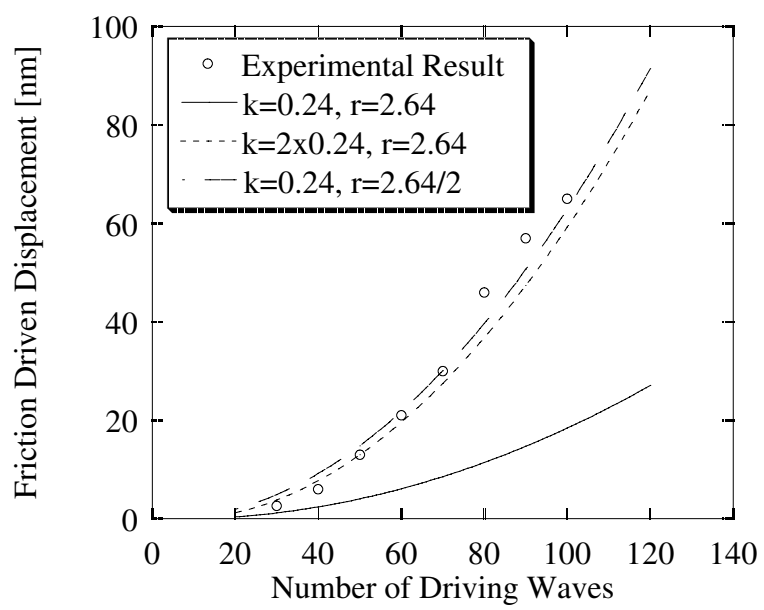

Fig. 10. The calculated friction driven displacement and the experimental result; the driving voltage was $100 V_{\text {peak }}$, the pre-load was $30 \mathrm{~N}$.

- $\mathrm{k}=0.24[\mathrm{~N} / \mathrm{nm}]$

- $\mathrm{r}=2.64[\mathrm{~N}]$.

The mass of the movable part and the steady state amplitude of the Rayleigh wave at $100 \mathrm{~V}_{\text {peak }}$ driving voltage were $10.31 \mathrm{~g}$ and $20.2 \mathrm{~nm}$. Substituting these numerical values to the equations (4) and (5) carried out the simulation. The calculated results are shown in Fig.10. The experimental results are also shown in this figure. Both, experimental and calculated, friction driven displacements increase with the square of the number of the driving waves, but the values are quite different. However, if substituting $2 \times k$ into $k$ or $r / 2$ into $r$, the difference between the calculated and experimental results are all within $5 \%$.

The calculated speed was compared with the experimental result in Fig.11, where the driving waves $N$ were 50 cycles. Both speeds are normarized by the maximum speed. There is a good agreement between the calculated and experimental results. They have same characteristics of

- linearlity of increasing their speed $(20 \leq \mathrm{n} \leq \mathrm{N})$

- quadratic functional saturation $(\mathrm{N} \leq \mathrm{n} \leq \mathrm{N}+20)$

- linearlity of decreasing their speed $(N+20 \leq n)$.

The characteristics indicate that the assumption, which the output force depends on the amplitude of the Rayleigh wave, was validated. 


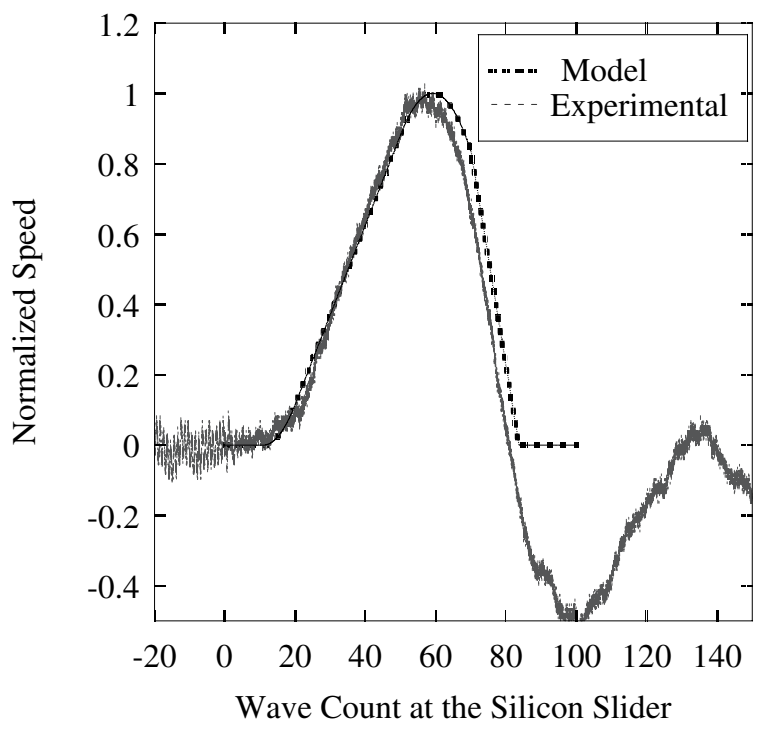

Fig. 11. The calculated speed and the measured speed of the silicon slider; the driving voltage was 100 $V_{\text {peak }}$, the driving waves $N$ were 50 cycles and the pre-load was $30 \mathrm{~N}$. They were both normalized by the maximum speed.

\section{DISCUSSION}

Figure 12 shows stepping drive motion in detail. This motion is the same motion of Fig.3, but it is enlarged to examine the detailed step motion. When the Rayleigh wave is excited, friction with surface particles of the stator transducer yields the driving force to the silicon slider. The friction driven displacement is defined in Fig.12, as the displacement during the Rayleigh wave is excited. It is the first part of the step motion and is the actual driven displacement due to the friction between the silicon slider and the surface particles of the Rayleigh wave.

The second part is where the driving voltage is not applied; the Rayleigh wave has passed away. There is no driving force from the wave to the silicon slider at this part, but extra displacement was yielded as seen in Fig.12. It was caused by the inertia of the linear guide stage. The mass of the linear guide stage was $9.42 \mathrm{~g}$, and that of the silicon slider with glued hemisphere was $0.89 \mathrm{~g}$. The mass of the linear guide stage was much larger and the friction with the linear guide rail was existed. Then the response of the linear guide rail was slower. Hence, the silicon slider (and the glued hemisphere) was dragged when the Rayleigh

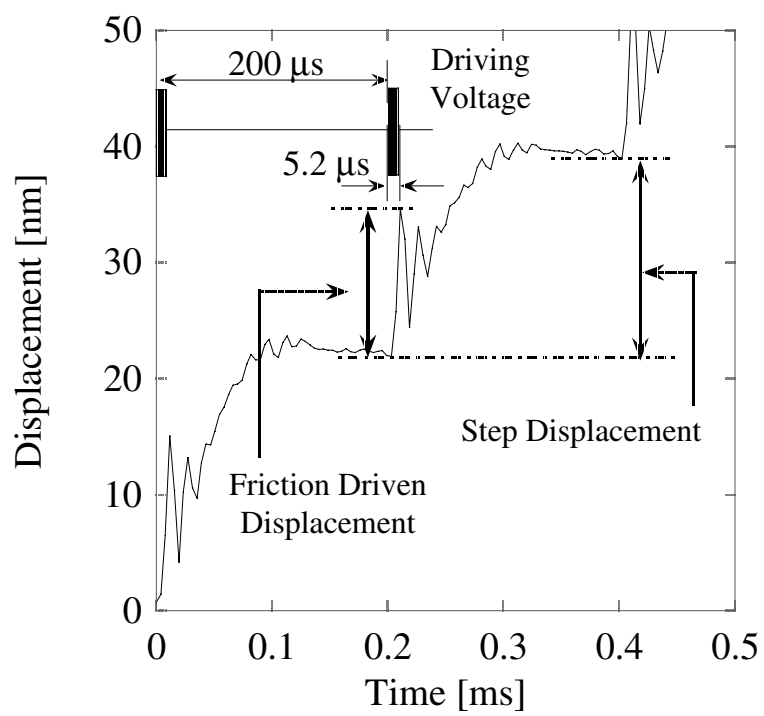

Fig. 12. Detail of the stepping drive; the driving voltage was $100 V_{\text {peak }}$, the driving waves were $50 \mathrm{cy}$ cles, and the pre-load was the $30 \mathrm{~N}$. The burst period was $0.2 \mathrm{~ms}$.

wave had passed away. It indicated that the movable part of the experimental setup did not move as a single mass. Namely, the movable part behaved as the two connected masses. One was the lighter slider part that consisted of the silicon slider and the steel hemisphere, and the other was the heavier linear guide stage part.

After the friction drive, vibration occurred as shown in Fig.12. This vibration happened at the connection of the movable part's two masses. The washer and the steel hemisphere were used for the connection of these two parts to make the silicon slider contact the stator transducer properly. The friction drive of the silicon slider made elastic deformation at this connected region during the first part of the step motion. The elastic deformation caused the followed vibration of the second part of the step motion.

The simplified mechanical model of the movable part is proposed in Fig.13 considering the compliance of the structure. The elastic connection between the steel hemisphere and the washer is indicated by the spring. When the Rayleigh wave is excited, the driving force is given to the silicon slider. The silicon slider is driven to the direction to compress the spring. The compressed spring thrusts the linear guide stage in the meanwhile. After the Rayleigh wave has passed away, there is no driving force from the wave to the silicon slider and 


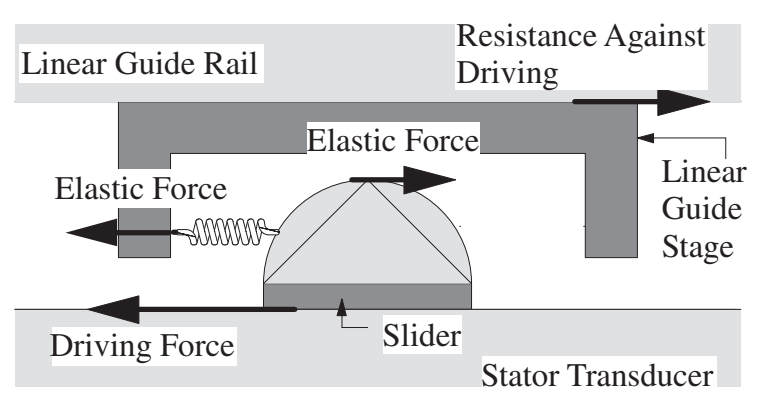

Fig. 13. The simplified mechanical model of the movable part.

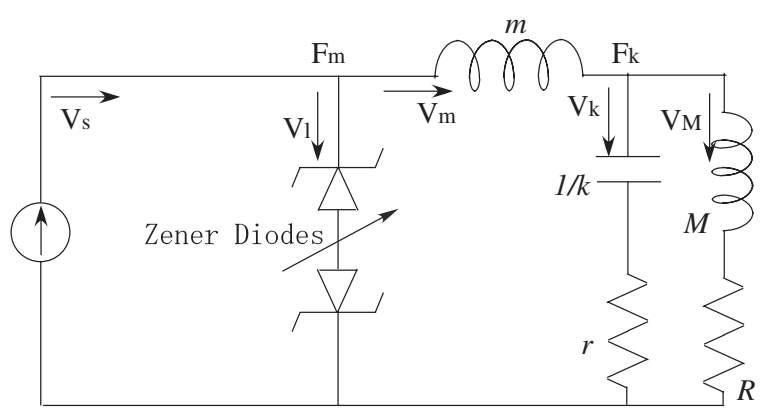

Fig. 14. The equivalent circuit model where the elastic deformation is taken into consideration.

then the silicon slider is thrust back by the elastic force of the compressed spring. In this way, the slider part, namely the silicon slider and the glued steel hemisphere, starts vibration according to the mass of the slider part and the elastic constant of the spring.

On the other hand, the linear guide stage is thrust by the inertia that is given by the first movement of the driven slider part. As previously indicated, the mass of the linear guide part was about 10 times larger than that of the slider part. Hence, the linear guide stage does not vibrate as the slider part does. The linear guide stage responds slower to the elastic force of the compressed spring.

The indicated simulation model considers that the movable part moves as a single mass, but it actually behaved as two connected masses. It is the reason of the difference between the calculated and experimental results in Fig.10. To simulate the dynamic behaviors of the movable part, the mechanical equivalent circuit model is proposed, as shown in Fig.14. $M$ and $m$ denote the mass of the linear guide stage and the mass of the slider part. $k$ and $r$ denote the elastic constants of the connection of the two masses. $R$ denotes the driving resistance of the linear guide stage. A pair of Zener diodes indicates the frictional force.

\section{CONCLUSION}

Changing the number of the driving waves was the easiest way to control the step displacement. In this way, the $2 \mathrm{~nm}$ stepping motion was obtained as the minimum step motion of the experimental setup. We also proposed the simulation model for open loop control of the step motion by changing the number of the driving waves. The model will simulate the more accurate dynamics of the movable part with taking the elastic deformation of the movable part into consideration.

\section{ACKNOWLEDGMENT}

This work was supported by the Grant-in-Aid for the Development of Innovative Technology of the Ministry of Education, Culture, Sports, Science and Technology of Japan.

\section{REFERENCES}

[1] S. Ueha and Y. Tomikawa, "Ultrasonic motorsTheory and Applications," Oxford, Clarendon Press, 1993.

[2] M. Kurosawa, M. Takahashi, and T. Higuchi, " Ultrasonic Linear Motor Using Surface Acoustic Waves," IEEE Trans. on Ultrason., Ferroelect., and Freq. Cont., Vol. 43, No. 5, pp. 901-906, 1996.

[3] M. K. Kurosawa and T. Higuchi, "Surface Acoustic Wave Motor," Proc. of 3rd Int. Heintz Nixdorf Symp., Paderborn, Germany, pp.113-118, 1999.

[4] M. K. Kurosawa, " State-of-art surface acoustic wave motor and its future applications," Ultrasonics 38, pp.15-19, 2000.

[5] M. K. Kurosawa, H. Itoh, K. Asai, M. Takasaki and T. Higuchi, " Optimization of Slider Contact Face Geometry for Surface Acoustic Wave Motor," Proc. of Micro Electro Mechanical Systems, Interlaken, Suisse, pp.252-255, 2001.

[6] K. Nakamura, M. Kurosawa, H. Kurebayashi, and S. Ueha, "An Estimation of Load Characteristics of an Ultrasonic Motor by Measuring Transient Responses," IEEE Trans. on Ultrason., Ferroelect., and Freq. Cont., Vol. 38, No. 5, pp.481-485, 1991. 